\title{
Detection of Cotton Leaf Diseases Using Image Processing
}

\author{
S. Batmavady, S. Samundeeswari
}

\begin{abstract}
Agriculture is the main occupation of the Indian population. Plant diseases are the alarming threat to the livelihood of agriculturists. Hence, proper detection and classification of the diseases will help the farmers to take precautionary measures and to increase the crop yield. The paper focusses on applying image processing and neural network techniques in identifying the diseased cotton leaves from the set of images taken from the village plant dataset. The leaves are first pre-processed to filter the noise and then converted to gray scale image. Image enhancement is done to sharpen the image followed by clustering and segmenting the diseased parts. Then, a set of features are extracted followed by the classification of the disease using Radial Basis Function (RBF) neural network classifier. Training and testing are carried out on a large number of samples and the results are compared with that of SVM classifier. The proposed method outweighs the SVM classification technique in providing better accuracy.
\end{abstract}

Index Terms: Classifier, Clustering, Image Enhancement, Pre-Processing, RBF Network, Segmentation, SVM.

\section{INTRODUCTION}

Cotton is an important crop of India contributing to the economy of India and plays a pivotal role in the agricultural sector. Agricultural research using technology is mainly focused on increasing the crop productivity, quality and the economic conditions of farmers. But, the productivity is hampered mainly due to environmental conditions and also due to the disease caused by fungus, bacteria and other pathogens. Manual identification of the diseases may sometimes happen to be erroneous due to fatigue and lighting conditions. Further, long time observation of the crops is essential for the accurate detection of the diseases and to take corrective measures. Instead, image processing could be employed to identify the diseases as early as possible and to plan and act accordingly. This helps in utilizing proper rectification measures followed by improved productivity and financial well-being of the famers.

Automatic disease detection and classification is a blooming research area in agricultural segmentation of diseased leaves using automation is a great boon to the farmers. K-means clustering technique is one such technique used for segmenting the diseased part of the leaf. Once the

Revised Manuscript Received on July 05, 2019

Dr.S.Batmavady, Professor in the department of ECE, Pondicherry Engg. College, Puducherry.

Ms.S.Samundeeswari, PG student of Pondicherry Engineering College, Puducherry. diseased part is segmented out, classification of the disease using Support Vector Machine classifier is done utilising

colour and texture features and their combinations[1]. If the diseases are identified at an appropriate stage, suitable corrective measures can be taken to avoid any further detrimentation to the crops. Pattern recognition method was employed in [2] to identify and classify the leaf diseases. Paper [3] focusses on segmentation and feature extraction employing colour co-occurrence matrix. Segmentation of diseased cotton leaves were carried out using Sobel and Canny edge detection techniques followed by HPCCDD classification method[4] . Identification of the plant disease is the key to preventing the losses in the yield and quantity of the agricultural product. Paper [5]discusses the method based on eigen spectrum modelling for the detection of plant disease using their leaves images. This paper [5] focusses on eigen feature regularisation and extraction approach to segment the diseased part of the leaf followed by feature extraction to extract the features of infected leaves to detect the diseases. Image processing techniques used in leaf disease detection are discussed in [6] and [7]. Paper [8] discusses the effective way used in the detection of plant diseases through leaf feature inspection. Health status of the plant is determined from the leaves.. Color features are extracted from the segmented output to differentiate the healthy and diseased samples. FCM clustering technique is used to segment the diseased leaf followed by SVM classification in paper [9]. A survey on the detection and classification of cotton leaf diseases is carried out in paper[10].Genetic algorithm is applied for the segmentation of diseases in the leaves of different plants [11] and SVM classifier was used in classification.

The proposed approach in the paper uses FCM clustering for segmentation followed by the extraction of more number of statistical features to improve the detection accuracy. Further, RBF neural network training is done on a huge number of data samples adding to improved performance.

Section II of the paper focuses on the proposed work of segmenting and classifying the diseased cotton leaves. Section III discusses the results of the proposed methodology and a comparison is made with that of SVM classifier. Section IV ends with conclusion and future scope of the work.

\section{PROPOSED WORK}

Input cotton leaf images are acquired from village plant dataset. Initially, the images are converted to gray scale followed by noise removal using median filter. Contrast of the image is improved using histogram enhancement in a pseudo colour image. Further, enhancement is achieved

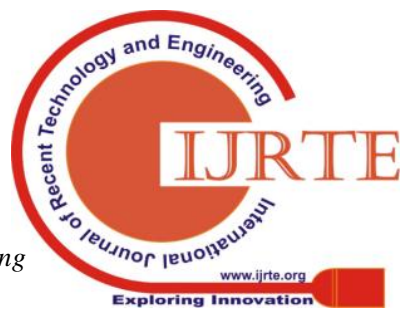


using morphological process. Fuzzy C-Means algorithm is used to segment the diseased part of the leaf. From the segmented output, twelve statistical features are extracted. These features are input to SVM classifier and also to the proposed RBF neural network classifier. Results show that the proposed method outweighs SVM classification in terms of accuracy, specificity and sensitivity. The proposed block diagram is as shown in Figure (1).

\section{RESULT}

The results of the proposed work are depicted in the following figures and comparison is made with the existing technique.

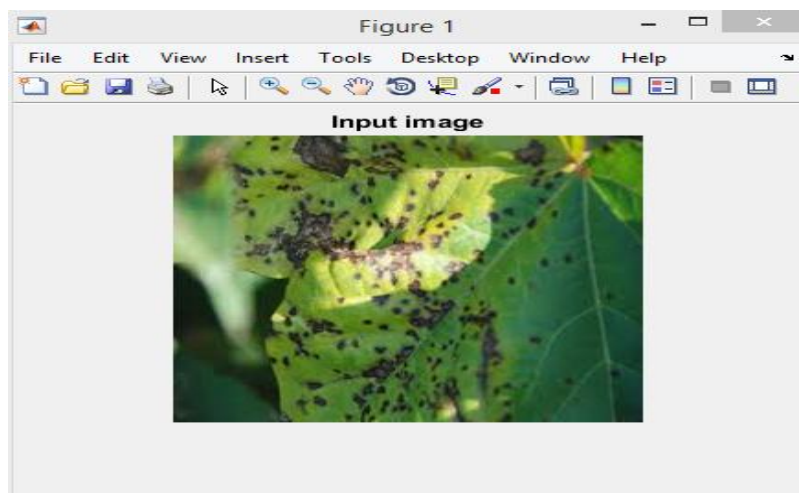

Figure(2) Input Image

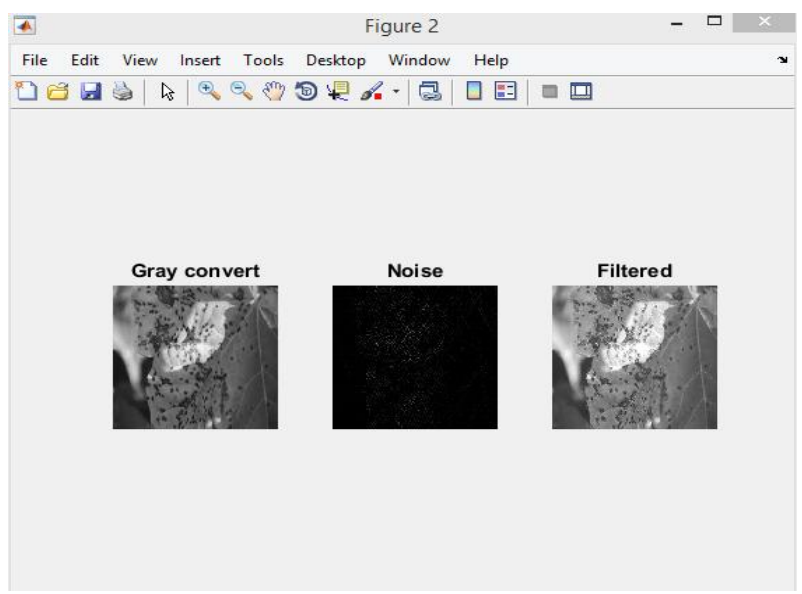

Figure(3) Pre-Processing

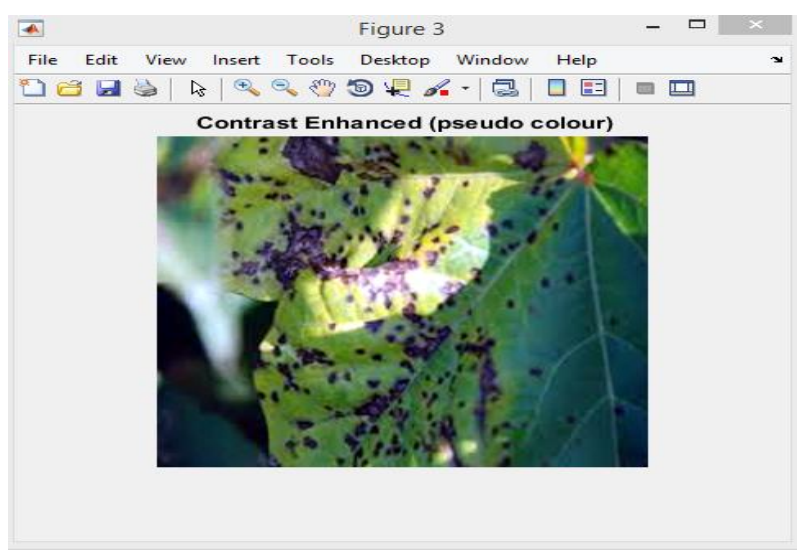

Figure (4) Enhancement

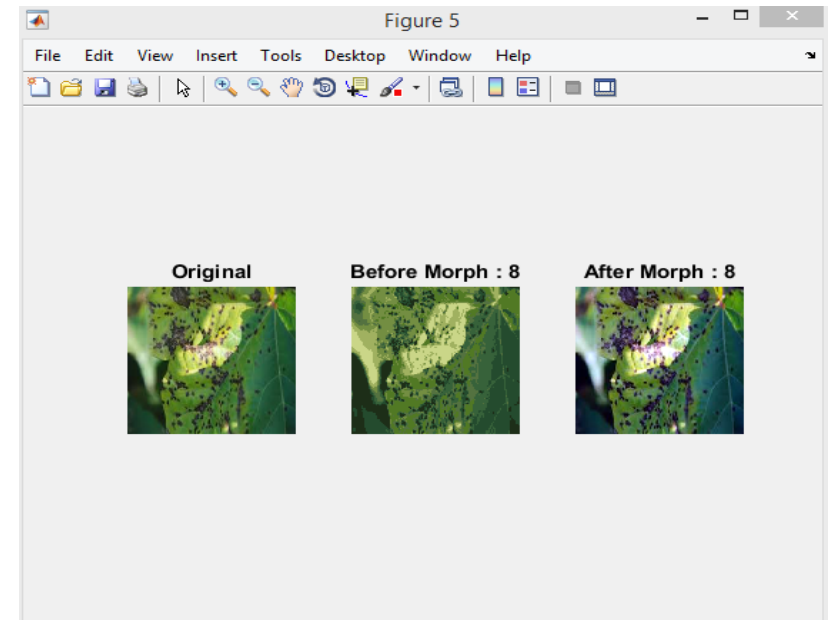

Figure (5) Morphological Processing

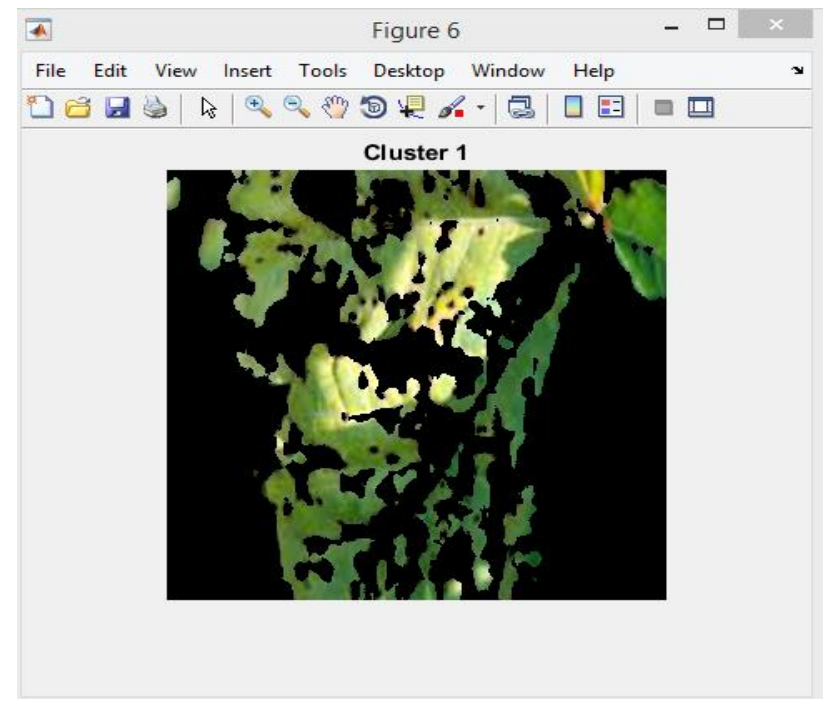

Figure (6) Cluster 1

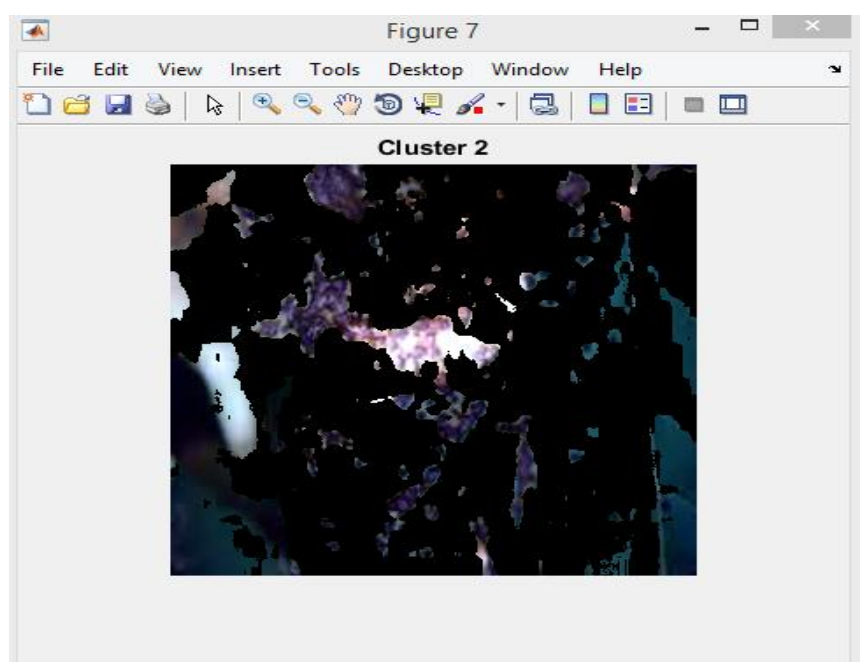

Figure (7) Cluster 2

Published By:

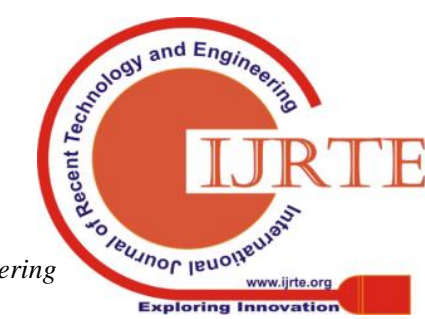




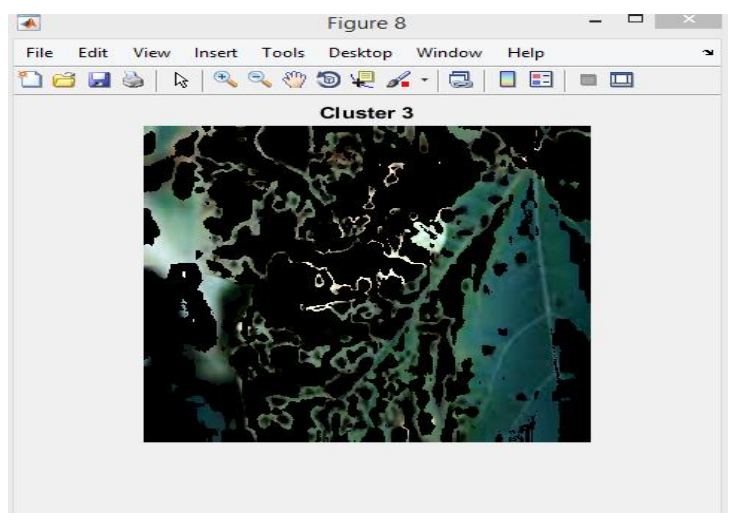

Figure (8) Cluster 3

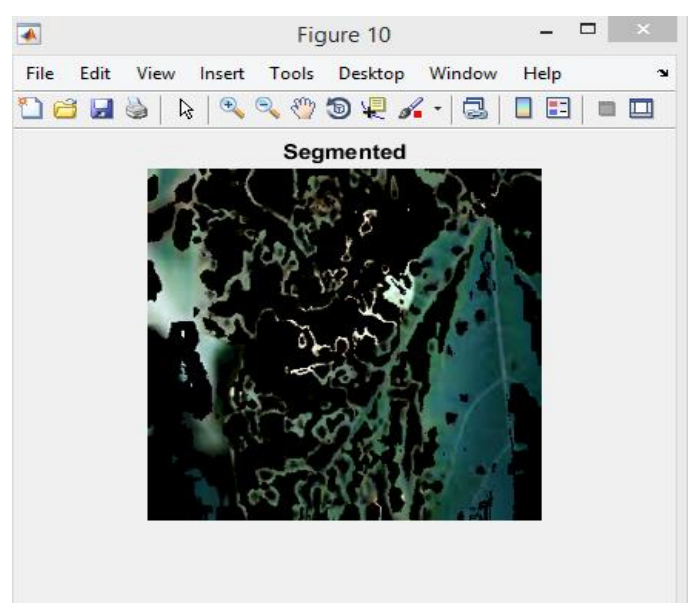

Figure (9) Segmented Output

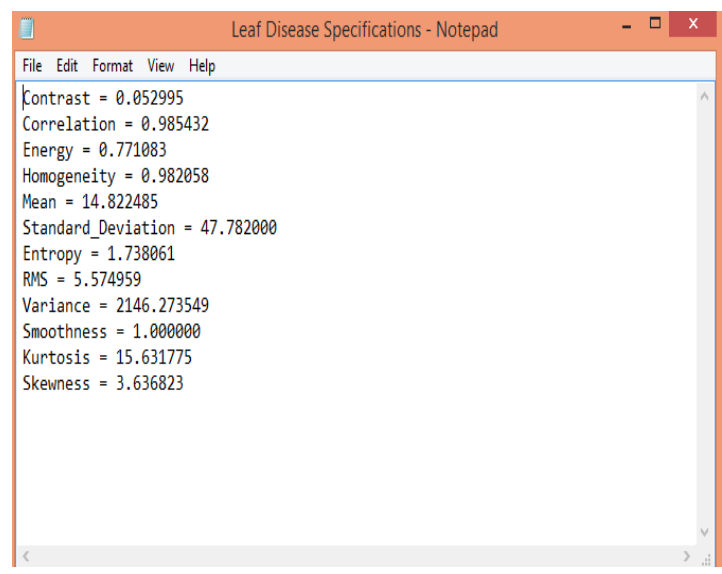

Figure (10) Feature Extraction

\section{Performance metrics}

Training Images - 290

Testing Images - 36

Elapsed Time for Training Images $-14.00 \mathrm{~s}$

Elapsed Time for Testing Images $-2.97 \mathrm{~s}$

No. of Input Nodes - 12
No. of Hidden Layers -3

No. of Output Node -1

\section{Statistical features of segmented image:}

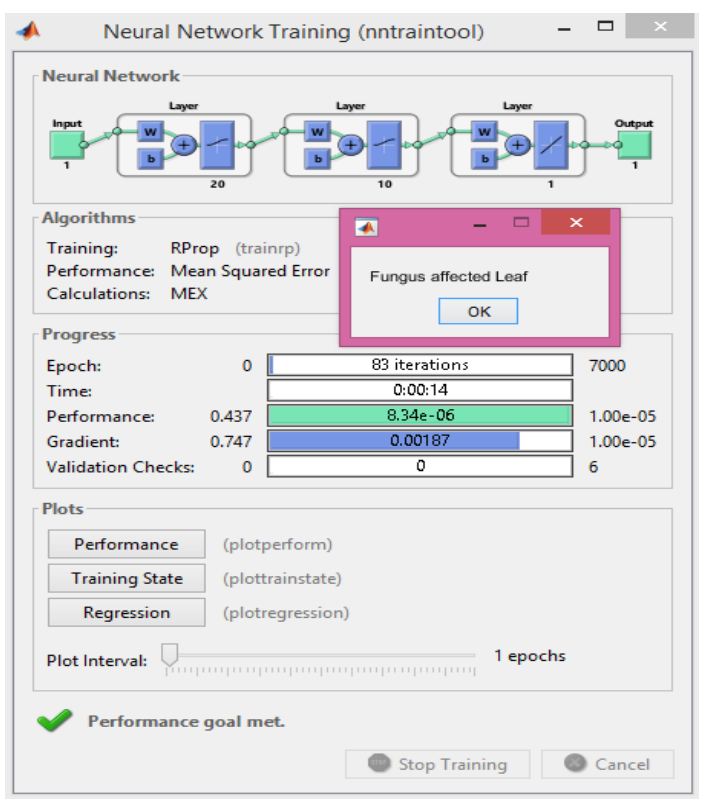

\section{Confusion Matrix:}

\section{(i) Existing System}

\begin{tabular}{|c|c|c|c|}
\cline { 2 - 3 } \multicolumn{1}{c|}{} & $\begin{array}{c}\text { PREDICTED } \\
\text { YES }\end{array}$ & $\begin{array}{c}\text { PREDICTED } \\
\text { NO }\end{array}$ & \multicolumn{1}{c}{} \\
\hline $\begin{array}{c}\text { ACTUAL } \\
\text { YES }\end{array}$ & TP=11 & FN=3 & $14(39 \%)$ \\
\hline $\begin{array}{c}\text { ACTUAL } \\
\text { NO }\end{array}$ & $\mathrm{FP}=2$ & $\mathrm{TN}=20$ & $22(61 \%)$ \\
\hline \multicolumn{1}{c|}{} & $13(36 \%)$ & $23(64 \%)$ & \multirow{2}{*}{$\mathrm{N}=36$} \\
\cline { 2 - 3 } & & &
\end{tabular}

(ii)Proposed system

\begin{tabular}{|c|c|c|c|}
\cline { 2 - 3 } \multicolumn{1}{c|}{$\mathrm{N}=36$} & $\begin{array}{c}\text { PREDICTED } \\
\text { YES }\end{array}$ & $\begin{array}{c}\text { PREDICTED } \\
\text { NO }\end{array}$ & \multicolumn{1}{c}{$13(36 \%)$} \\
\hline $\begin{array}{c}\text { ACTUAL } \\
\text { YES }\end{array}$ & $\mathrm{TP}=12$ & $\mathrm{FN}=1$ & $23(64 \%)$ \\
\hline $\begin{array}{c}\text { ACTUAL } \\
\text { NO }\end{array}$ & $\mathrm{FP}=1$ & $\mathrm{TN}=22$ & \\
\hline & $13(36 \%)$ & $23(64 \%)$ & \multirow{2}{*}{$\mathrm{N}=36$} \\
\cline { 2 - 3 } & & & \multicolumn{2}{c}{} \\
& & &
\end{tabular}

Sensitivity $=\frac{\mathrm{TP}}{(\mathrm{TP}+\mathrm{FN})}$

Accuracy $=\frac{(\mathrm{TN}+\mathrm{TP})}{(\mathrm{TN}+\mathrm{TP}+\mathrm{FP}+\mathrm{FN})}$

Specificity $=\frac{\mathrm{TN}}{[\mathrm{FP}+\mathrm{TN})}$ 


\begin{tabular}{|l|l|l|}
\hline PARAMETERS & $\begin{array}{l}\text { EXISTING } \\
\text { WORK }\end{array}$ & $\begin{array}{l}\text { PROPOSED } \\
\text { WORK }\end{array}$ \\
\hline Sensitivity & $83.33 \%$ & $92.00 \%$ \\
\hline Accuracy & $85.44 \%$ & $90.00 \%$ \\
\hline Specificity & $95.00 \%$ & $96.00 \%$ \\
\hline
\end{tabular}

\section{Block Diagram of the proposed work:}
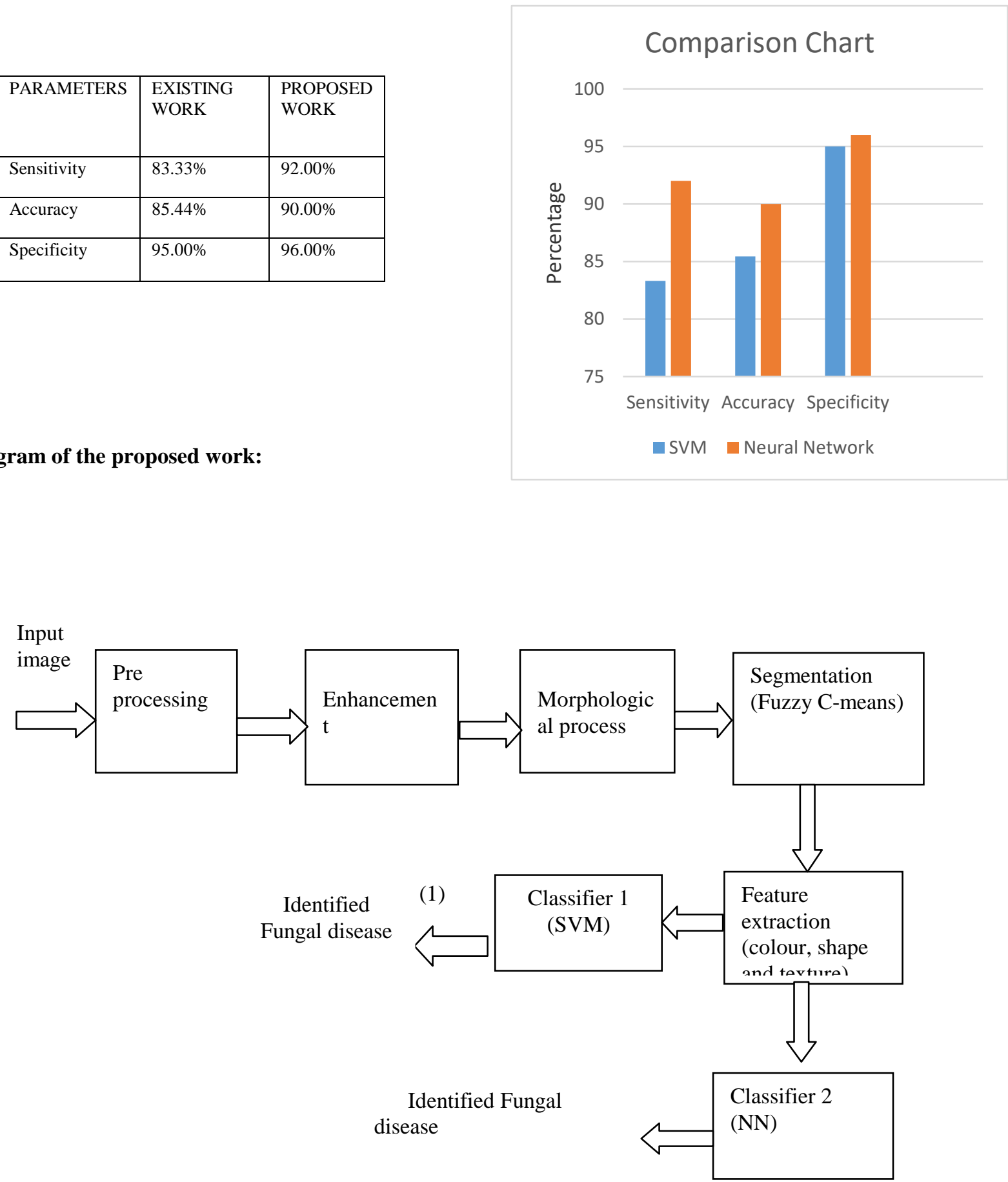

\section{CONCLUSION}

The diseased cotton leaves are segmented using FCM technique after pre-processing. From the segmented output, the extracted features are used to classify the disease in the leaf using RBF neural network. The proposed method is found to be superior compared to SVM classification. Future work may be focused on real time implementation of the proposed algorithm for continuous monitoring and detection of plant diseases 


\section{REFERENCES}

[1] Sukhvir Kaur, Shreelekha Pandey, Shivani Goel, "Semi-automatic leaf disease detection and classification system for soybean culture," IET Image Process, vol. 12, issue. 6, pp. 1038-1048, May 2018.

[2] P. R. Rothe, Dr. R. V. Kshirsagar, "Cotton leaf disease identification using pattern recognition techniques," International Conference on Pervasive Computing (ICPU), vol. 74, issue 1, pp. 267-275, July 2015.

[3]Sanjay B. Dhaygude, Mr. Nitin P. Kumbhar, "Agricultural plant leaf disease using image processing," International Journal of Advanced Research in Electrical Electronics and Instrumentation Engineering, vol. 2, issue 1, ISSN 2278-8875, January 2013.

[4] P. Revathi, M. Hemalatha, "Classification of cotton leaf spot diseases using image processing edge detection techniques," IEEE: International Conference on Emerging Trends in Science, Engineering and Technology, vol. 2, issues 5, pp. 3661-3664, August 2012

[5] Ajay A. Gurjar, Viraj A. Gulhane, "Disease detection on cotton leaves by eigen feature regularization and extraction technique," International Journal of Electronics Communication \& Soft Computing Science and Engineering, vol. 1, issue 1, pp. 1-4, June 2011.

[6] Sachin D.Khirade, A. B Patil, "Plant Disease Detection using Image Processing," International Conference on Computing Communication Control and Automation, vol. 4, issue 2, pp. 453-455, May 2014.

[7] M. Malathi, K. Aruli, S. Mohamed Nizar, A. SagayaSelvaraj, “A Survey on Plant Leaf Disease Detection using Image Processing Communication Control and Automation, vol. 4, issue 2, pp. 453-455, May 2014.

[8] Malvika Ranjan, Manasi Rajiv Weginwar, Neha Joshi, Prof. A. B Ingole, "Detection and Classification of Leaf Disease usingArtificial Neural Network," International Research Journal of Engineering and Technology (IRJET), vol. 2 issue 9, pp. 578-580, August 2015.

[9] S.Megha, C.R.Niveditha and etal., "Image Processing System for Plant Disease Identification by using FCM clustering Technique," International Journal of Advanced research, Ideas and Innovations in Technology, vol. 3, 1 issue 2, , pp. $445-449,2017$.

[10] Bhumika S. Prajapati, Vipul K. DabhiHarshadkumar, B. Prajapati, "A Survey on Detection and Classification of Cotton Leaf Disease," International Conference on Electrical Electronics and Optimization Techniques (ICEEOT), vol. 7, issue 12, pp. 55-60, June 2016.

[11] Vijai Singh a, * , A.K. Misra ," Detection of Plant leaf Diseases using Image Segmentation and Soft Computing Techniques", Information Processing in Agriculture, pp. 41-49, 2017

\section{AUTHORS PROFILE}

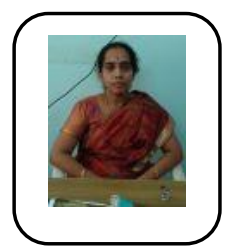

Dr.S.Batmavady is currently working as Professor in the department of ECE, Pondicherry Engg. College, Puducherry. Her area of specialization is Digital Image Processing. She has published quite a good number of papers in many journals and conferences.

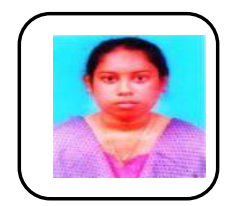

Ms.S.Samundeeswari is the PG student of Pondicherry Engineering College, Puducherry. She completed her graduation in Pondicherry 